\title{
Virtual magnetic resonance elastography has the feasibility to evaluate preoperative pituitary adenoma consistency
}

\author{
Kerstin Lagerstrand ${ }^{1,2}$ (1) $\cdot$ Nicholas Gaedes $^{3}$. Stig Eriksson ${ }^{4} \cdot$ Dan Farahmand $^{5,6}$ - Erica De Coursey ${ }^{4}$. \\ Gudmundur Johansson ${ }^{7,8} \cdot$ Lars Jönsson $^{4} \cdot$ Thomas Skoglund $^{5,6}$
}

Accepted: 19 January 2021 / Published online: 8 February 2021

(c) The Author(s) 2021

\begin{abstract}
Purpose To evaluate the use of preoperative virtual Magnetic Resonance Elastography (vMRE) for patients undergoing transsphenoidal resection of pituitary adenomas (PA).

Methods Ten patients $(60.2 \pm 19.6$ years; 8 males) were prospectively examined with the vMRE-method prior to transsphenoidal surgery. vMRE-images, reflecting tissue stiffness were reconstructed. From these images, histograms as well as the mean stiffness values over the tumor body were extracted. Finally, vMRE-data was compared with the PA consistency at surgery blinded to vMRE.

Results In all patients, successful vMRE-examination was performed enabling evaluation of even small PAs. For tumors with homogenous tissue, the mean stiffness value increased with surgical consistency grading. For heterogenous tumors, however, the mean stiffness value did not consistently reflect the grading at surgery. On the other hand, the vMRE-images and histograms were found to be able to characterize the tumor heterogeneity and display focal regions of high stiffness that were found to affect the surgery outcome in these PAs. The vMRE-images and histograms showed great promise in characterizing the consistency at surgery for these PAs.

Conclusion Evaluation of PA consistency in preparation for surgery seems to be feasible using the vMRE-method. Our findings also address the need for high resolution diagnostic methods that can non-invasively display focal regions of increased stiffness, as such regions may increase the difficulty of transsphenoidal PA-resection.
\end{abstract}

Keywords Magnetic resonance imaging $\cdot$ Virtual elastography $\cdot$ Pituitary macroadenoma $\cdot$ Consistency $\cdot$ Stiffness

Kerstin Lagerstrand

kerstin.lagerstrand@vgregion.se

1 Medical Physics and Biomedical Engineering, Sahlgrenska University Hospital, Göteborg, Sweden

2 Institute of Clinical Sciences, Sahlgrenska Academy, University of Gothenburg, Göteborg, Sweden

3 Philips, Göteborg, Sweden

4 Department of Radiology, Sahlgrenska University Hospital, Göteborg, Sweden

5 Department of Neurosurgery, Sahlgrenska University Hospital, Göteborg, Sweden

6 Department of Clinical Neuroscience and Rehabilitation, Institute of Neuroscience and Physiology, The Sahlgrenska Academy, University of Gothenburg, Göteborg, Sweden

7 Department of Medicine, Sahlgrenska University Hospital, Göteborg, Sweden

8 Department of Internal Medicine and Clinical Nutrition, Institute of Medicine, The Sahlgrenska Academy, University of Gothenburg, Göteborg, Sweden

\section{Introduction}

Pituitary adenomas (PA) account for 10-15\% of all intracranial tumors $[1,2]$. The majority of the PAs have a soft texture and can be resected with curettage and suction [3]. However, about $10 \%$ of the tumors have a fibrous content that makes the tumor difficult to completely resect without injury to surrounding vital structures [4]. Preoperative knowledge of the PA consistency may alter the surgical technique and have an impact on the surgical approach and outcome, as well as reduce the risk for re-surgery or the need for post-surgical adjuvant radiotherapy [5, 6].

To characterize the consistency of PAs, various magnetic resonance imaging (MRI) methods based on standardized T1- and T2-weighted imaging, as well as contrast media enhanced MRI imaging and standardized diffusion weighted imaging have been investigated [6-10]. Up to this date, these methods have not shown sufficient feasibility 
in characterizing the PA consistency. Magnetic resonance elastography, however, which relies on the propagation of mechanically induced shear waves [11-13], has shown to display stiffness values that correlate with surgical grading [14]. While being a promising method for evaluation of PA consistency, the method lacks in image resolution and this may limit the visibility of small tumors and focal regions of increased tissue stiffness in larger tumors. Also, the method relies on additional hardware for induction of brain vibrations.

In this exploratory study, a novel method is proposed. Besides being a non-invasive method that relies on a clinically available MRI sequences with high speed and image resolution, this so called virtual elastography (vMRE) method is attractive for evaluation of the PA consistency due to an intrinsically high sensitivity to the viscoelastic property of the tissue. To our knowledge, however, the method has not previously been used for tumor evaluation.

Therefore, the aim of this study was to evaluate the potential usefulness of the vMRE method for preoperative evaluation of tumor consistency in patients undergoing transsphenoidal resection of PA.

\section{Methods}

\section{Study cohort}

The study was conducted according to the Declaration of Helsinki. Ethical approval was given by The Regional Ethics Review Board and oral and written informed consent was obtained from all participants. The data was derived from the Gothenburg Pituitary Tumor (GoPT) study, which is a prospective study that is enrolling patients scheduled for pituitary surgery at Sahlgrenska University Hospital, the sole provider of neurosurgical services for 1.8 million people in the western region of Sweden [15].

The patients were included among patients referred to the radiology department from 17/10/2018 to 31/03/2020, where patients with macroadenoma $(\geq 1 \mathrm{~cm})$ who underwent an MRI the day before surgery for neuro-navigation, was included in the study. The indications for surgery were (1) tumor causing chiasmal compression and/or (2) hormonal hypersecretion and/or (3) sequential imaging showing growth of the tumor.

The tumor type was verified by histology.

\section{Magnetic resonance imaging}

All patients were examined on a 3T MRI scanner (Achieva, Philips Medical systems, The Netherlands). A standardized preoperative examination protocol, including morphological imaging in the sagittal view and standardized angiography in the transversal view (Table 1) was used in addition to diffusion weighted imaging. The full examination protocol was completed within $20 \mathrm{~min}$, where the diffusion weighted imaging was completed in less than $6 \mathrm{~min}$.

\section{The vMRE method}

The diffusion weighted imaging sequence was included in the examination protocol to characterize the viscoelastic property of the tissue using the vMRE method. For that purpose, higher b-factors $\left(b=200\right.$ and $\left.1000 \mathrm{~s} / \mathrm{mm}^{2}\right)$ were used. With the use of higher $b$-values, the normally high sensitivity to isotropic gaussian diffusion was shifted toward non-isotropic non-gaussian diffusion and thereby, the diffusion weighted imaging sequence was made sensitive to fibrotic tissue (see le Bihan et al. [16] for more thorough theoretical description). To increase the contrast-to-noiseratio and the acquisition speed of the diffusion weighted imaging, a fat-suppressed sequence based on compressed sensing (compressed SENSE reduction factor $=2$ ) was used. Also, the sequence was based on turbo spin echo readout module to reduce geometric distortions in the sellar region from bone air cavities in the sinuses.

\section{vMRE stiffness quantification}

After scanning, two diffusion weighted stacks of images were automatically generated on the scanner; one for the lower $b$-value (b200 images) and one for the higher $b$-value (b1000 images). These images were then used to quantitate vMRE stiffness values. The quantification is summarized below and described in detail by le Bihan et al. [16]. In short, a stack of vMRE stiffness images was automatically

Table 1 The MRI examination protocol

\begin{tabular}{|c|c|c|c|c|c|c|}
\hline Sequence name & Orientation & Field-of-View $\left(\mathrm{mm}^{2}\right)$ & Scan matrix & $\begin{array}{l}\text { Slice thick- } \\
\text { ness }(\mathrm{mm})\end{array}$ & $\begin{array}{l}\text { Number of } \\
\text { excitations }\end{array}$ & $\begin{array}{l}\text { Number } \\
\text { of slices }\end{array}$ \\
\hline 3D TOF angiography & TRA & $160 \times 160$ & $356 \times 357$ & 0.7 & 1 & 200 \\
\hline 3D morphological T1-weighted imaging & SAG & $250 \times 250$ & $250 \times 250$ & 1 & 1 & 183 \\
\hline 2D diffusion weighted imaging & COR & $220 \times 186$ & $124 \times 88$ & 3 & 5 & 13 \\
\hline
\end{tabular}

$2 D$ and $3 D$ two and three dimensional, TOF time-of-flight, TRA transversal, COR coronary, SAG sagittal 
calculated from the stack of b200 and b1000 images as the relative difference between the signal values in the b200 and b1000 images, respectively.

$v M R E$ stiffness $=-9.8 \ln \left(S_{b 200} \mid S_{b 1000}\right)+14$,

where $S_{\mathrm{b} 200}$ and $S_{\mathrm{b} 1000}$ are the signal values in the b200 and b1000 images and the scaling factor of -9.8 and the shift factor of 14 were previously determined in a calibration step by le Bihan et al. [16].

Then, the tumor region was segmented on all vMRE stiffness images that included the tumor body. The border of the segmentation was located approximately one pixel away from the visible edge of the tumor, ensuring that no background tissue was included in the tumor region (Fig. 1). Also, pixels in major blood vessels were excluded from the region. The segmentation was performed manually by two trained observers (E.D.C and N.G), unaware of the surgical findings, and took approximately $5 \mathrm{~min}$ per individual. N.G repeated the segmentations to determine the intra-observer agreement. The inter-observer agreement between the observers was also determined.

From the segmented tumor regions, the mean vMRE stiffness and the standard deviation of the mean were automatically extracted within $1 \mathrm{sec}$. Also, histograms were automatically extracted to visualize the distribution in vMRE stiffness values within the tumor region.

The quantification of the vMRE stiffness was performed off-line using MATLAB R2016a (Mathworks, Natick, Massachusetts, USA).

\section{The PA consistency at surgery}

All surgeries were performed by one of two surgeons (D.N. or T.S.). The surgeons, blinded to the vMRE stiffness images, gave a single classification of tumor stiffness in each case as very soft, soft, moderate, hard or very hard (I-V). The system for classification of the consistency was jointly developed by the surgeons and has systematically been performed as a part of the prospective GOPT-study since 2015. In addition, the surgeons also made separate notes about the consistency of the tumor in free text.

\section{Descriptive and statistical analysis}

Categorical variables were expressed by numbers and continuous variables by mean $\pm \mathrm{SD}$.

The vMRE stiffness images and histograms, as well as the quantitative parameters (mean vMRE stiffness and the standard deviation of the mean) were compared with surgical findings as well as between individuals.

The Chi-squared test was conducted to compare vMREvalues and ADC-values between surgical gradings at $p=0.05$ significance level.

The inter-observer agreement was determined using intraclass correlation coefficients (ICC) with $95 \%$ confidence intervals, where the two-sided random ICC model was used to determine the consistency of the agreement.

The statistical analyses were performed using the MatLab software.

\section{Results}

\section{Patient characteristics and PA consistency at surgery}

Ten patients $(60.2 \pm 19.6$ years; 8 males $)$ underwent preoperative MRI examination before endoscopic transsphenoidal resection of PA. The patient characteristics and results on PA consistency at surgery are shown in Table 2. The patients displayed a large range of tumor size from 10 to $44 \mathrm{~mm}$ and a large variation in PA consistency from very soft to very hard. Results from 6-months post-op follow-up, included when available, showed large regions of tumors residuals in two patients (ID $=1$ and 7). Also, in patients 2 and 3 , small regions of tumor residuals were found at 6-months post-op follow-up.

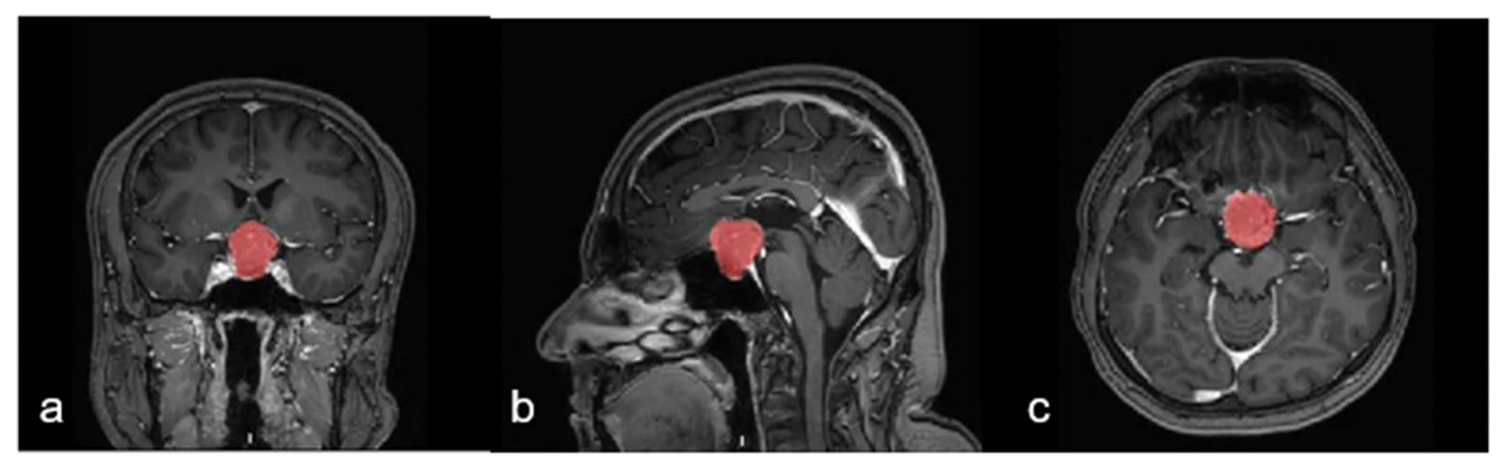

Fig. 1 Illustration of the tumor ROI on T1-weighted images in the coronal, sagittal and transversal plane 


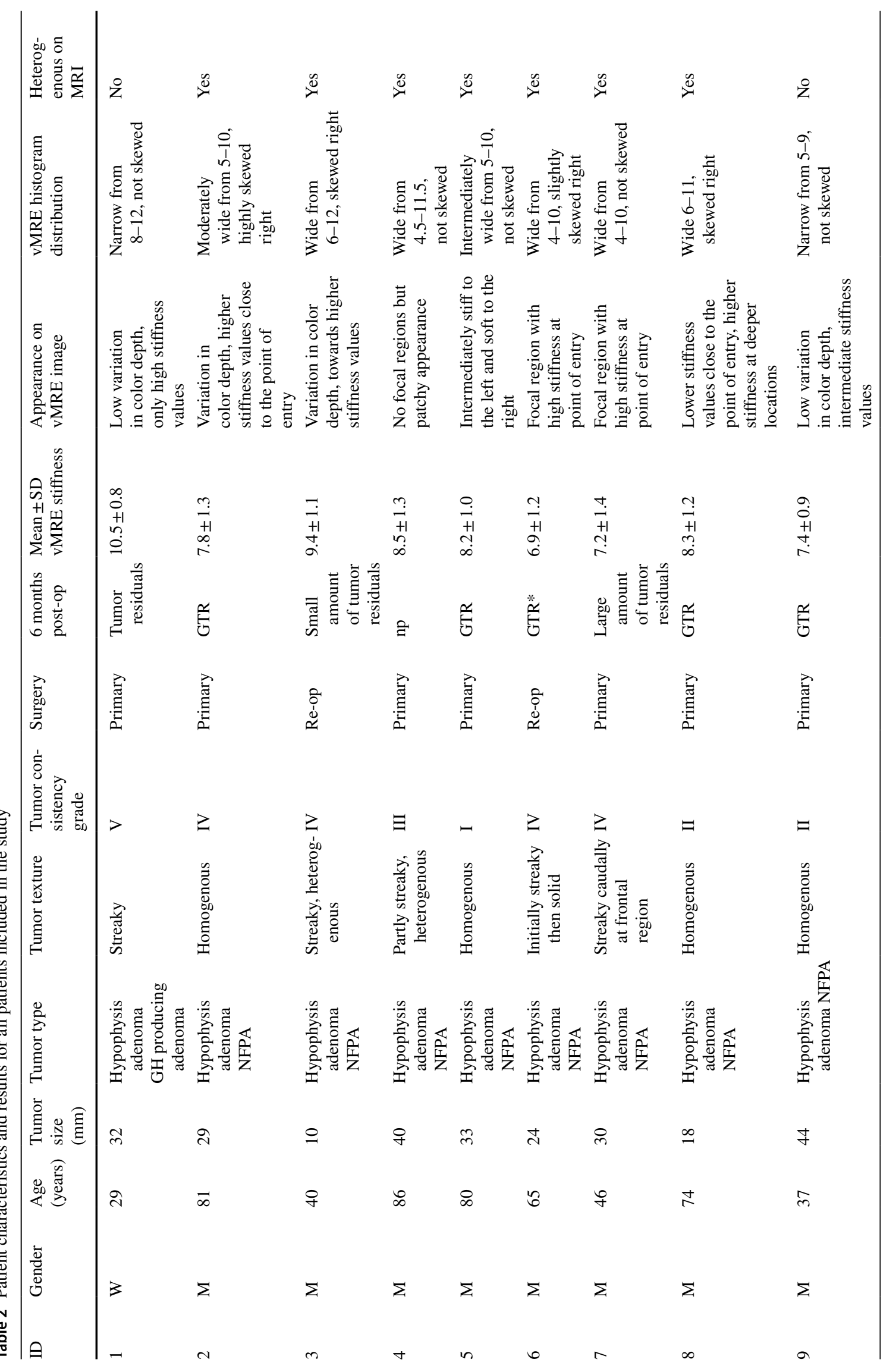




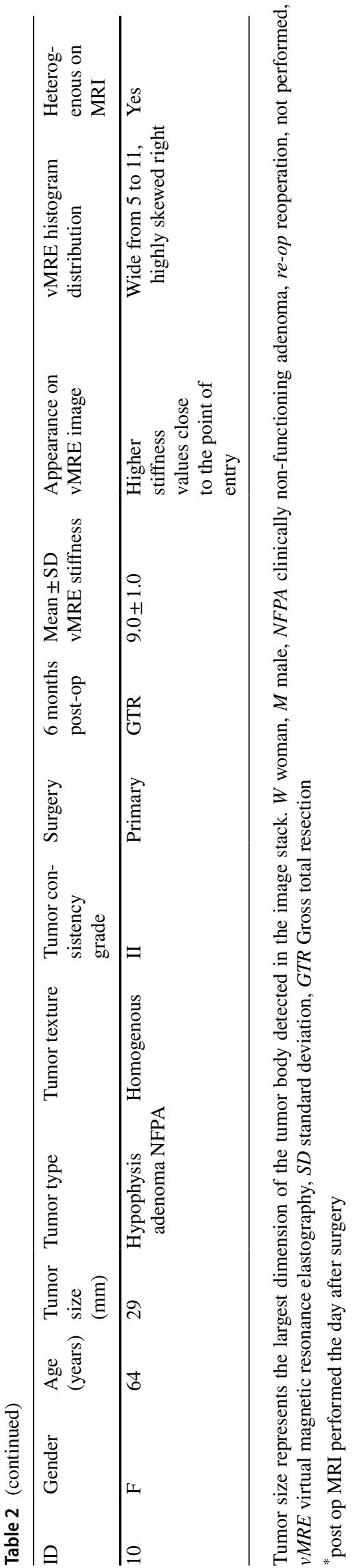

\section{Quantification of vMRE stiffness values}

The diffusion weighted imaging was successfully performed in all patients, resulting in vMRE stiffness images with high image quality.

Overall, the mean vMRE stiffness did not differ between groups of patients with different grading of PA consistency at surgery (Table 2). However, the mean vMRE stiffness value increased with increased grading for homogenous PAs (Fig. 2), displaying low color depths in the vMRE stiffness images and more narrow histogram distributions.

Between but also within individuals, the PAs displayed a large variation in vMRE stiffness values. Some PAs displayed focal regions of increased tissue stiffness, depicted as clusters of pixels with higher values in the vMRE stiffness images. The histograms characterized such heterogeneity as right-skewed distributions while streaky tissue was characterized in histograms as a large distribution of stiffness values. For example, ID 6 that was re-operated showed a region of higher stiffness values around 8.5 in the lower part of the tumor (close to the point of entry of the surgery) while deeper into the body, the tumor appeared soft with low stiffness values around 4 (Fig. 3). The corresponding histogram visualized the tumor heterogeneity as a large spread in stiffness values and a slightly right-skewed distribution. The histogram of ID 8 also displayed a heterogeneous PA with a right-skewed distribution of stiffness values. The corresponding vMRE stiffness image of ID 8 displayed a PA with low stiffness values around 4 close to the point of entry of surgery, while small regions of higher stiffness values, around 9, were found deeper into the tumor body (Fig. 4). In this case, the PA was graded as soft at surgery and showed gross total resection on post-op MRI. Both the vMRE stiffness image and histogram for ID 3 displayed the PA tissue as stiff and heterogeneous (Fig. 5), in similar with the PA consistency grading and tumor texture (Table 2). For ID 4, the vMRE stiffness images displayed the PA as intermediately stiff with a wide variation between image slices in the anterior posterior direction from high to low stiffness values (Fig. 6). The corresponding histogram also displayed a wide distribution of stiffness values. This PA was found to be soft/ moderate by the consistency grading (Table 2). For ID 2, the PA appeared to be very heterogenous in the vMRE stiffness images and showed a right-skewed histogram with a large proportion of high stiffness values. At surgery, the PA was graded as hard but showed gross total resection on post-op MRI (Fig. 7). See supplementary figures for remaining PAs.

The ICC analysis showed excellent agreement $(r=1.00$ and $p<0.001$ ) with near identical mean vMRE stiffness values in all three cases (E.D.C, N.G1, N.G2). 


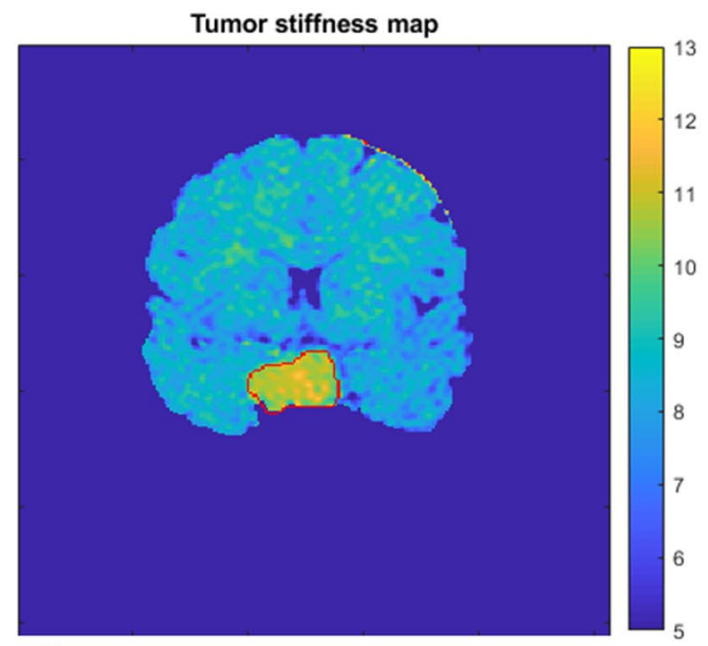

a

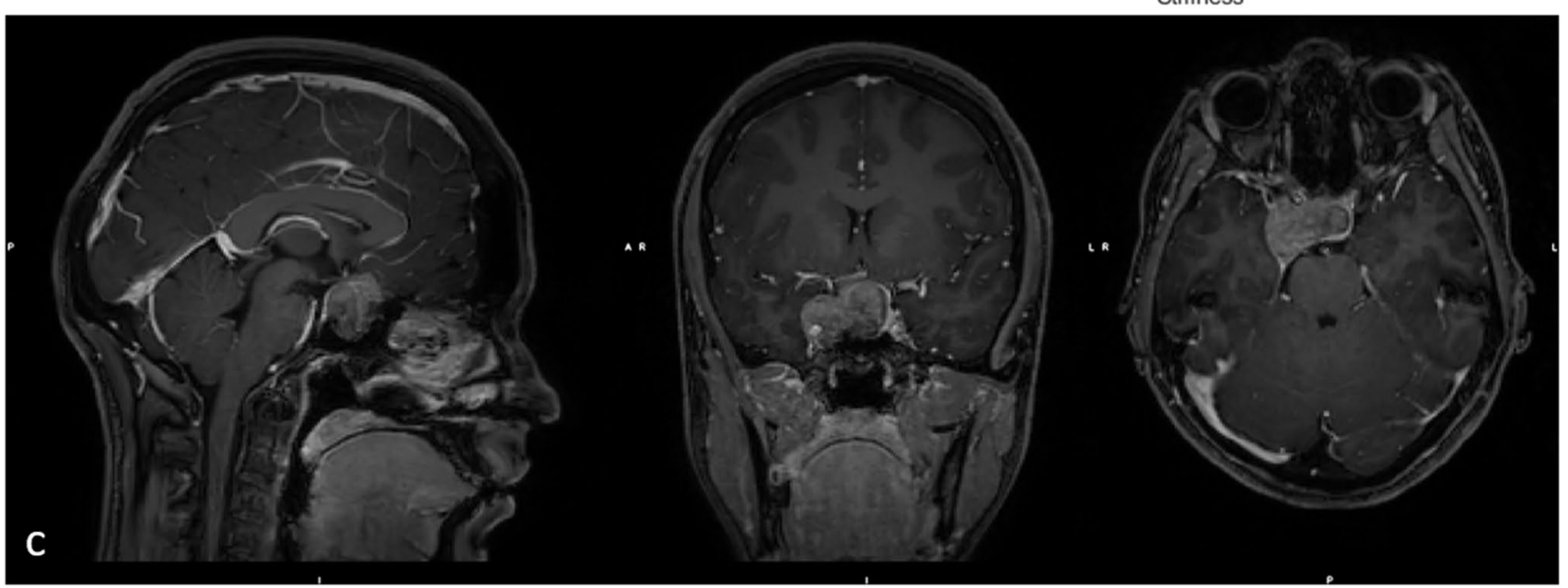

Tumor stiffness histogram

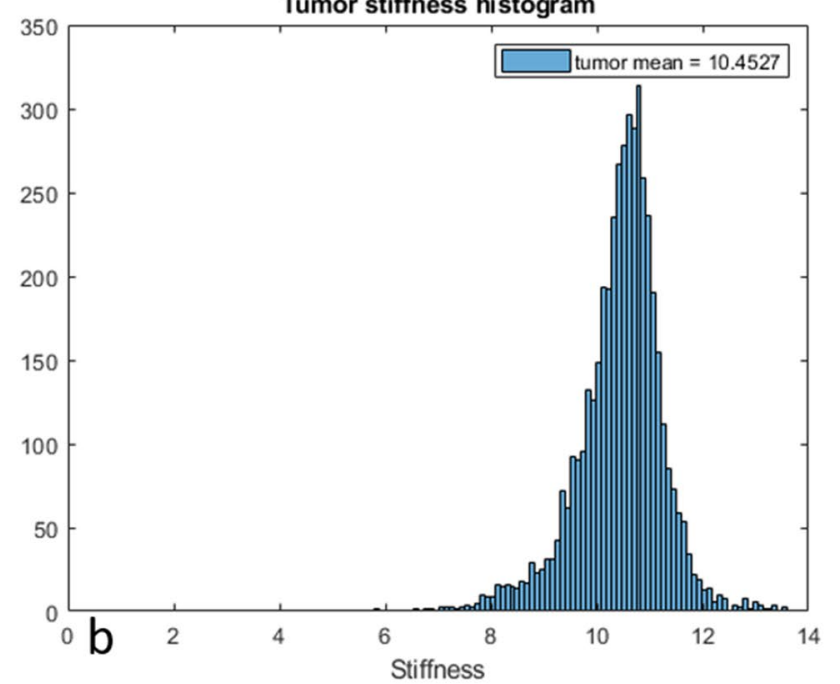

gery, very high stiffness with low variation over the tumor body

\section{Discussion}

In this study, a non-invasive diagnostic method for evaluation of PA consistency is proposed that has the potential to improve the risk assessment and surgical planning of these patients. For homogenous tumors, the so called vMRE method showed, in consensus with surgery grading, increased mean stiffness values with increased PA consistency. Moreover, stiffness values displayed in color in vMRE stiffness images and histograms greatly improved the tumor characterization, emphasizing focal regions of increased tissue stiffness that may pose a problem during surgery.

While most PAs have soft texture and can be resected easily, a small proportion are hard and are more difficult to dissect from critical surrounding structures. Such cases may add substantial amount of operative time and may demand further surgical methods with associated risks. Improving the preoperative prediction may lead to better counseling of the risks and benefits for the patient. Hence, new diagnostic tools are warranted for preoperative evaluation of PA consistency.

Several studies have used a variety of MRI-based methods to find correlations between image characteristics and PA consistency. Contrast media enhanced MRI has been used, without success, to differentiate soft from hard tumors using intratumorally hyperintense dots [10]. Also, no correlation between $\mathrm{T} 2$-weighted image characteristics and PA consistency has been found [17]. A few studies have correlated standardized diffusion weighted imaging to PA consistency $[8,18]$, but found that the calculated diffusion value is not predictive of tumor consistency [19]. This might, to some part, be assigned to perfusion that inherently contaminates the diffusion value. Since perfusion can vary greatly between tumors and, thus, introduce individual biases in the measurement, the method is not suitable for evaluation of underlying viscoelastic property of the PA tissue. Moreover, 

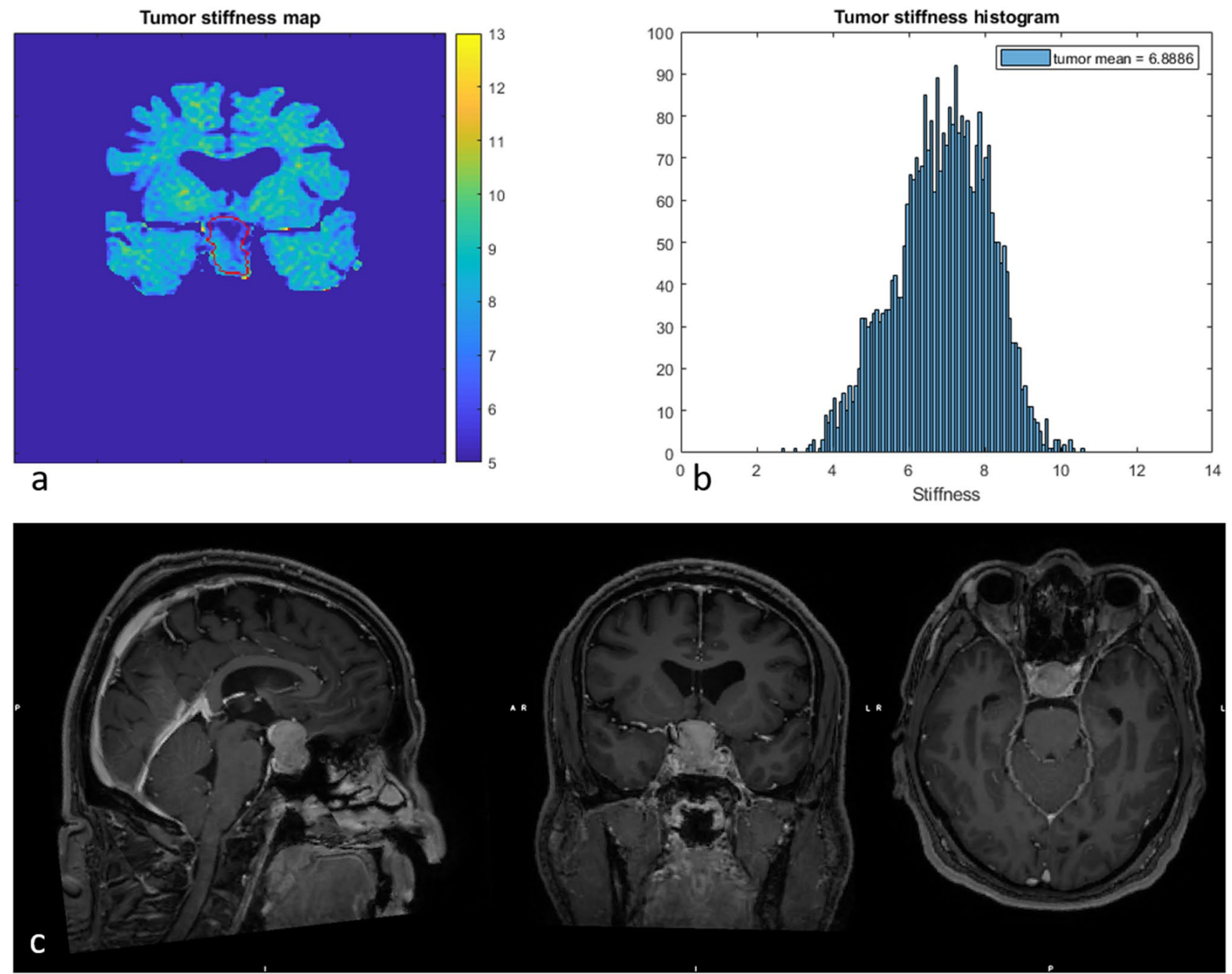

Fig. 3 The vMRE stiffness image (above left) for PA with ID 6 displayed a region of much higher stiffness values in lower part (close to the point of entry of the surgery) compared with the upper part of the tumor body. Corresponding histogram (above right) enhanced the tumor heterogeneity as a large spread in stiffness values and a slightly skewed distribution

diffusion. Non-Gaussian diffusion is believed to arise from diffusion barriers, such as cell membranes in fibrotic tissue. Hence, vMRE method could potentially provide a better understanding of microstructural tissue changes in PAs associated with disease pathology and enable fast and detailed preoperative evaluation of the tumor stiffness.

A recently published study has utilized standardized magnetic resonance elastography to determine PA consistency at surgery, where a correlation between the stiffness values and consistency grading was reported [14]. As in present study, the included sample size was small $(n=10)$ but the results were encouraging, suggesting that stiffness have the possibility to predict PA surgery outcome. The considerably higher resolution, but also the shorter scan time and availability of the proposed method makes the proposed method an attractive alternative to the standardized magnetic resonance 

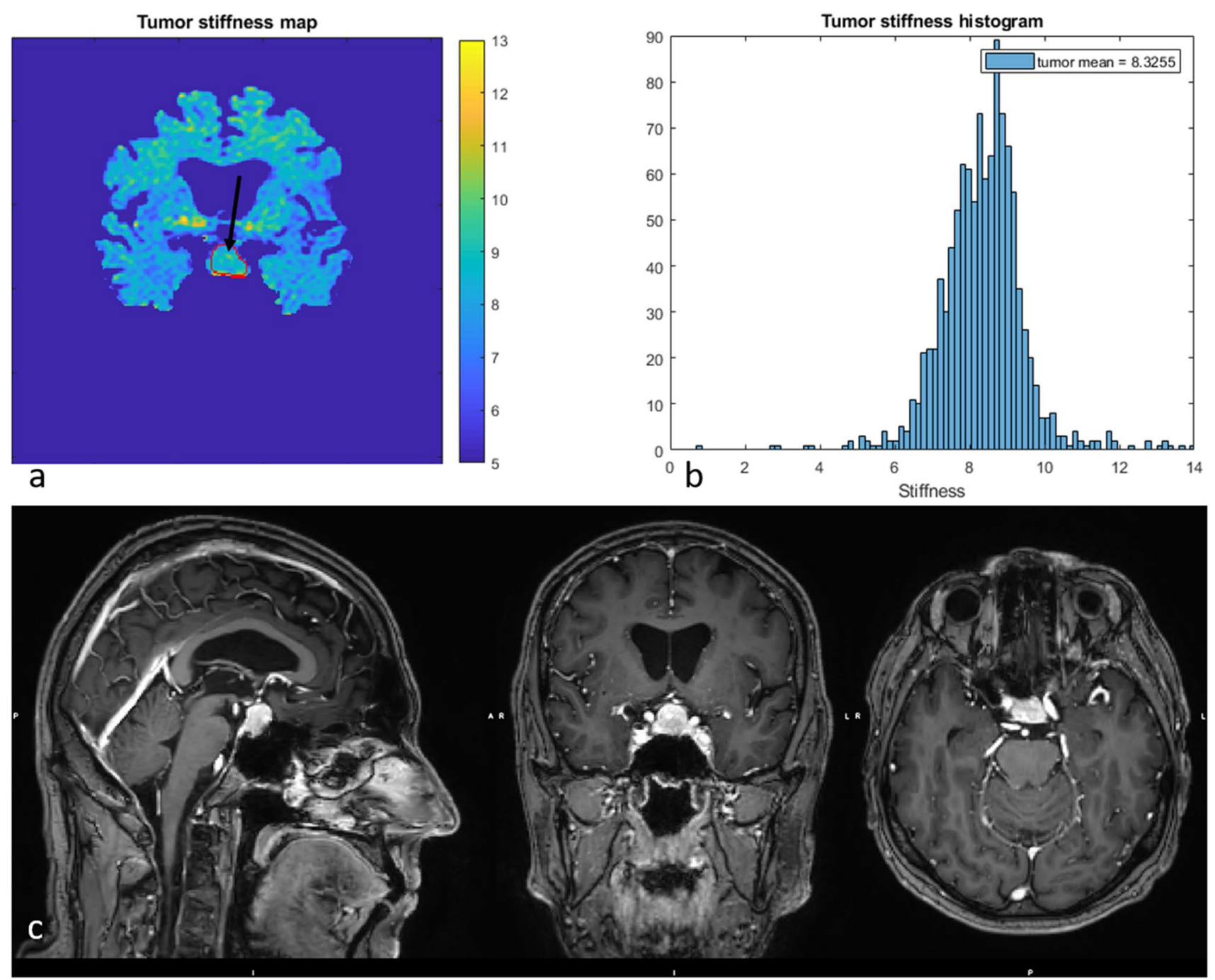

Fig. 4 The vMRE stiffness image (above left) for PA with ID 8 displayed a region of higher stiffness values deeper into the tumor body (black arrow) and lower stiffness values close to the point of entry.

elastography method for evaluation of PA consistency. Also, the present method does not rely on additional hardware for induction of measurable shear wave vibrations.

In similar to the standardized magnetic resonance elastography method, the vMRE method seemed to offer mean stiffness values that increased with surgical grading for homogenous tumors. The high-resolution vMRE method also offered detailed information about the tumor tissue in terms of color vMRE stiffness images and histograms, where our findings showed the importance of characterizing tumor tissue heterogeneity in detail. Focal regions of tissue with higher stiffness may alter the surgical approach and have an impact on the surgical outcome. For example, one of the PA patients (ID 6) was re-operated and as a result displayed a region of very stiff values due to scarring, clearly displayed in the vMRE stiffness images at the point of surgical entry.
Corresponding histogram (above right) enhanced the tumor heterogeneity as a slightly skewed distribution. The high stiffness values at the very bottom outside the tumor outline are likely bone

This might have limited the removal of the tumor. Preoperative knowledge of the position of the focal region might have altered the course of the surgery in this subject. Future studies are encouraged to confirm the diagnostic value of the vMRE method and the clinical usefulness of this novel stiffness contrast.

Since the proposed method has not previously been used for patients undergoing transsphenoidal resection of PAs, there is a lack of knowledge regarding the appearance of the PA tumors in the vMRE stiffness images and histograms, associated to the surgeon's experience at surgery. For homogenous PAs, there seems to be a consistency between the mean vMRE stiffness value and the surgery grading. For heterogenous tumors, however, deeper knowledge seems to be needed, as exemplified by ID 4. While the consistency of most PAs was found to be 

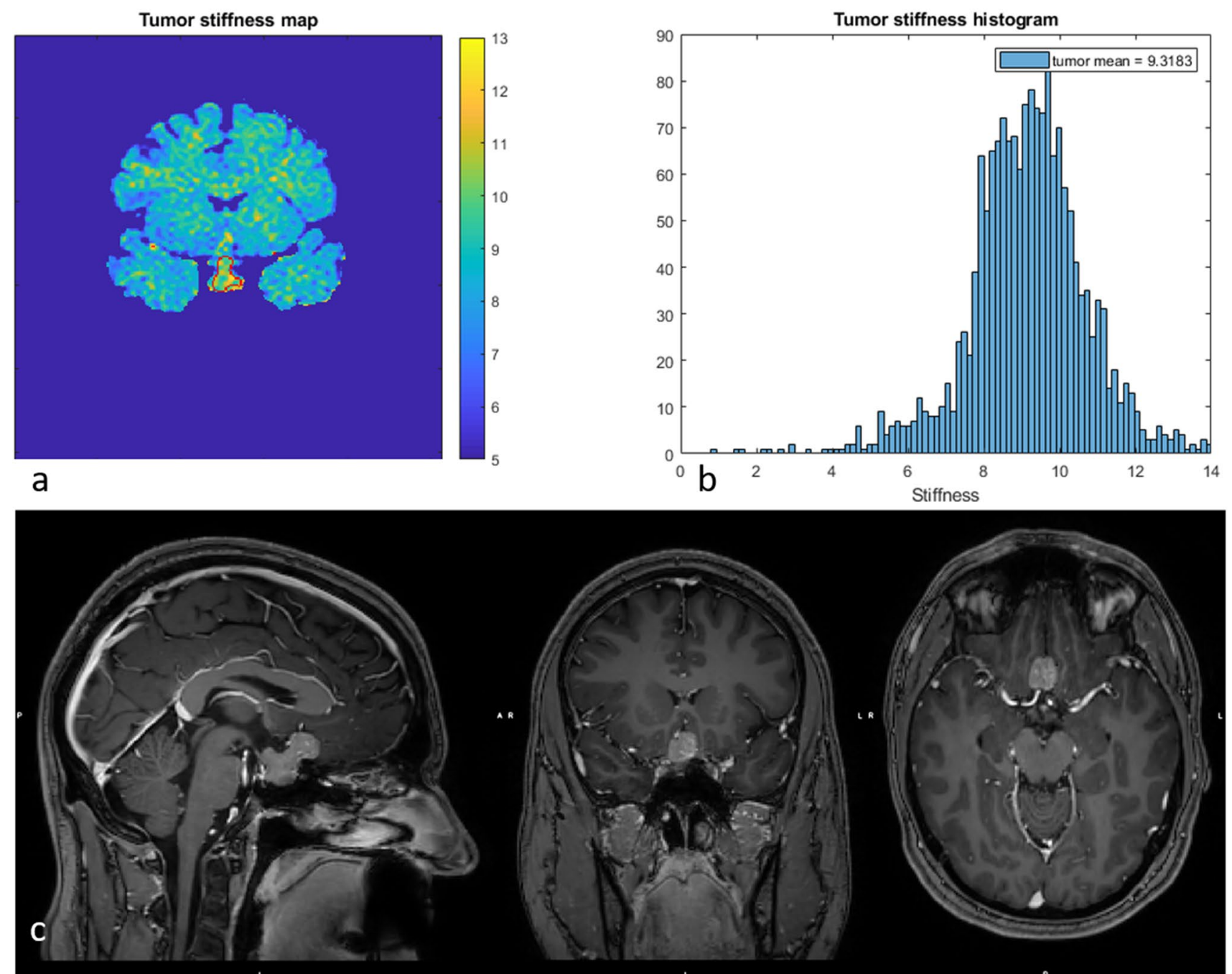

Fig. 5 The vMRE stiffness image (above left) and histogram (above right) for PA with ID 3 displayed a stiff and very heterogeneous tissue texture with a skewed histogram and a wide variation of stiffness values over the tumor body

successfully characterized with the present method, this softer PA displayed a mean vMRE stiffness value in similar to PAs with high consistency grading. No focal regions of higher stiffness values were seen in the vMRE stiffness images. However, the histogram displayed a wide distribution of stiffness values, representing a patchy heterogenous texture pattern. Such texture pattern might give a soft experience at surgery.

\section{Limitations}

Being a feasibility study, a small number of patients were included in the evaluation and, as such, this limits the strength of the conclusion. Future investigations on larger study cohorts and different types of adenomas are therefore encouraged to confirm our findings and evaluate the predictive value of the vMRE method for surgical PA outcome.

The proposed vMRE-method utilized a diffusion weighted imaging sequence that was sensitized to enhance tissue textures with dense diffusion barriers, such as fibrotic tissue. For that purpose, high $b$-values were used. Even though present findings show great promise, we cannot neglect the fact that the vMRE-method might have benefitted from even higher $b$-values, however, at the expense of increased spatial resolution or extended scan time.

In similar to the work by Hughes et al. [14], the study design was standardized so that each case was assigned a single classification of surgical impression of PA consistency, 

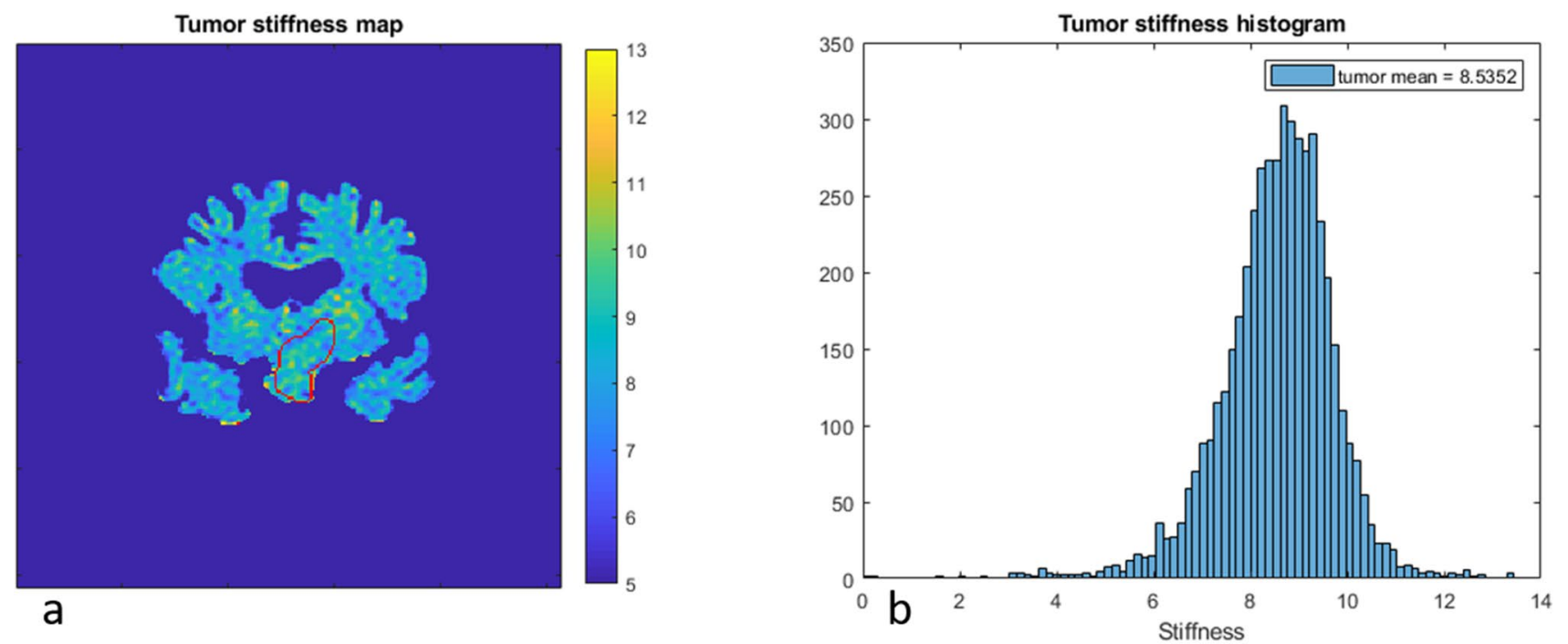

a

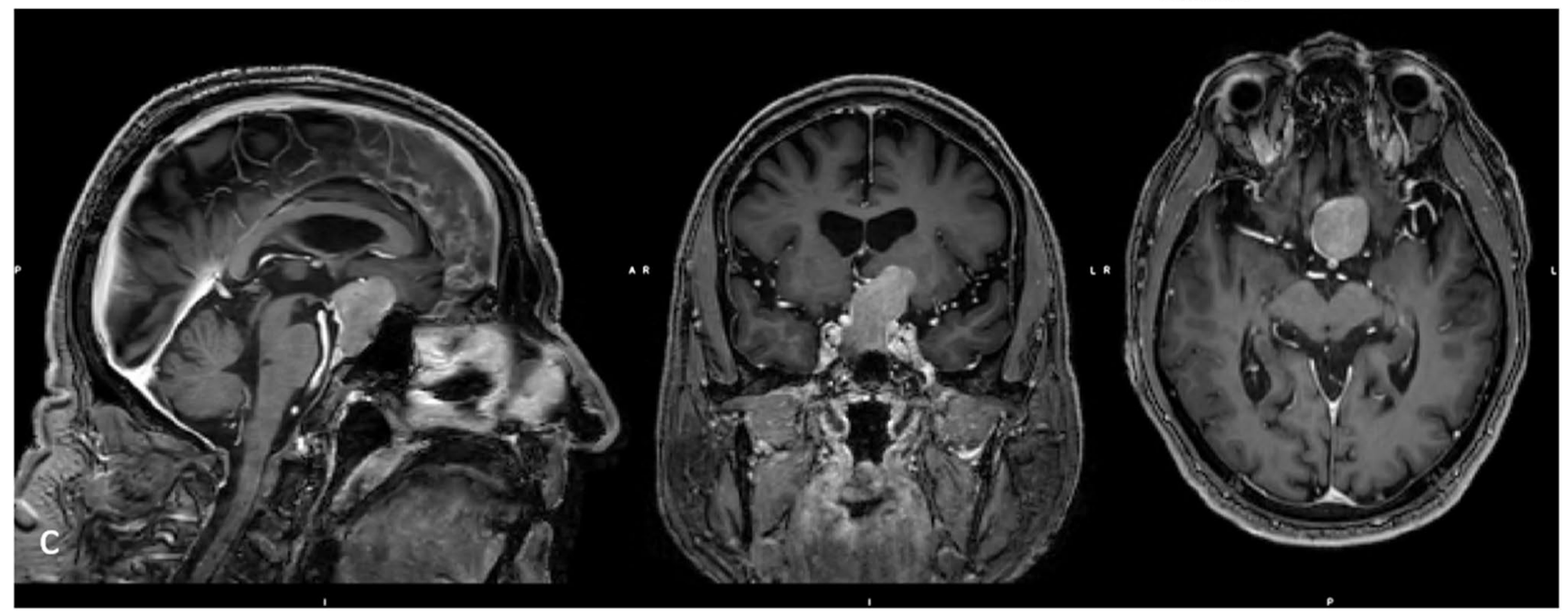

Fig. 6 The vMRE stiffness image (above left) and histogram (above right) for PA with ID 4 displayed a wide variation between image slices in the anterior posterior direction from high to low stiffness values. The chosen slice shows a stiffer part of the tumor

using a 5-point scale from very soft to very hard. However, the vMRE method seems to be able to characterize tumor heterogeneity in detail and our findings show the importance of including such information in the PA evaluation. To fully investigate the diagnostic value of the vMRE method, future studies should preferably include the surgeon's impression of heterogeneity in the classification protocol.

The vMRE stiffness calculation used in this study is straightforward, where the mean tumor stiffness can be estimated directly from the mean signals of the b200 and b1000 images. Future MR scanner releases may implement the proposed method and offer automatic evaluation. Today, however, an off-line software is needed to calculate the vMRE stiffness on a pixel by pixel basis and reconstruct vMRE stiffness images and histograms for PA heterogeneity evaluation. This may limit the evaluation in centers with no technical/programming competence.

\section{Conclusions}

Present study showed high feasibility of the vMRE method for preoperative evaluation of the PA consistency. The vMRE-method produced artifactual-free images of high quality and resolution, enabling evaluation of tumor heterogeneity. Also, the mean vMRE stiffness value was found to increase with surgical stiffness grading for solid tumors with homogenous texture. Further studies are encouraged to confirm our findings and determine whether the vMRE method provides useful information in the preoperative planning of PAs.

Supplementary Information The online version of this article (https:// doi.org/10.1007/s11102-021-01129-4) contains supplementary material, which is available to authorized users. 


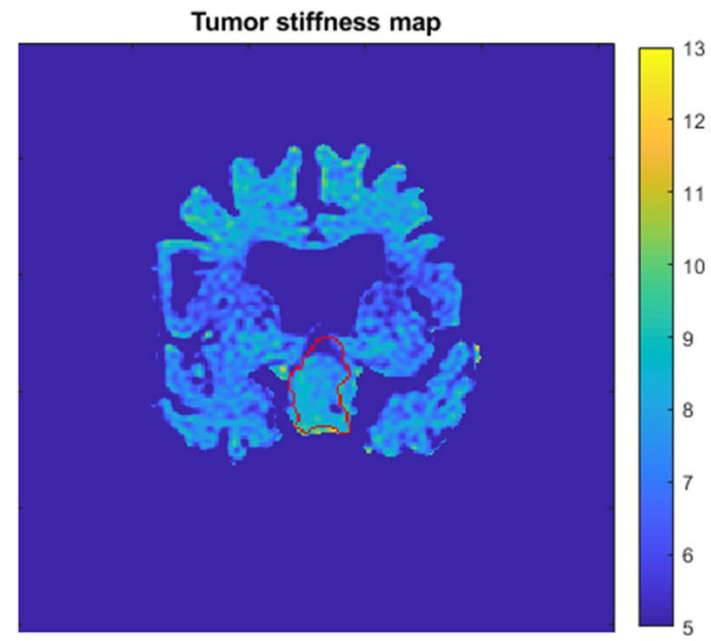

a
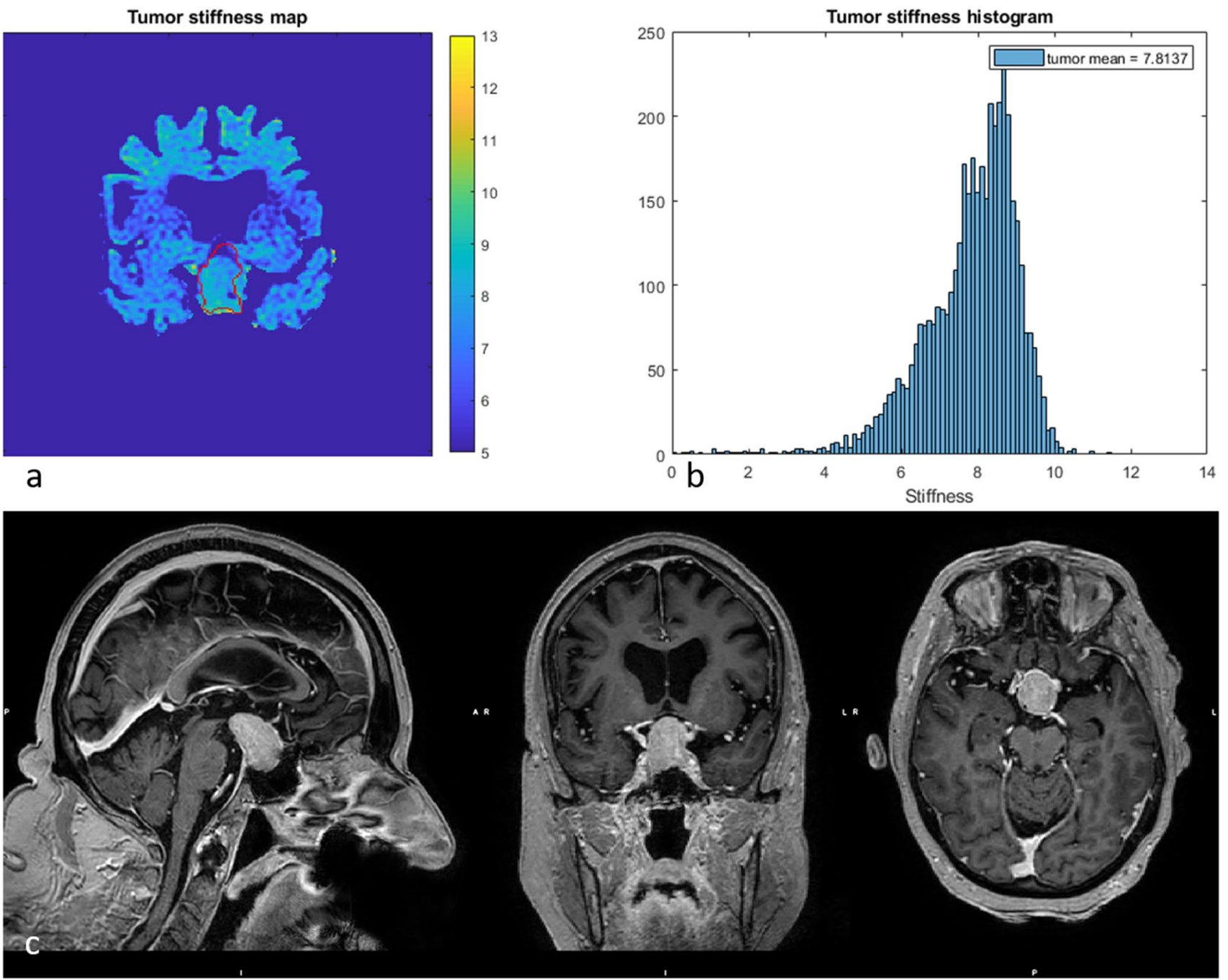

Fig. 7 The vMRE stiffness image (above left) and histogram (above right) for ID 2 showed a highly skewed histogram with a large proportion of high stiffness values, especially in the lower part of the

Funding The study was financed by Grants from the Swedish state under the agreement between the Swedish government and the country councils, the ALF-agreement.

\section{Data availability All images are available on request.}

Code availability The off-line post-processing software code is available on request.

\section{Compliance with ethical standards}

Conflict of interest The authors have nothing to disclose.

Ethical approval All procedures performed in studies involving human participants were in accordance with the ethical standards of the institutional and/or national research committee and with the 1964 Helsinki declaration and its later amendments or comparable ethical standards. tumor body where the incision was made. Interestingly, the very top part of the tumor is shown to be very soft

The study was conducted according to the Declaration of Helsinki. Ethical approval was given by The Regional Ethics Review Board.

Informed consent Oral and written informed consent was obtained from all participants before entering any part of the study.

Open Access This article is licensed under a Creative Commons Attribution 4.0 International License, which permits use, sharing, adaptation, distribution and reproduction in any medium or format, as long as you give appropriate credit to the original author(s) and the source, provide a link to the Creative Commons licence, and indicate if changes were made. The images or other third party material in this article are included in the article's Creative Commons licence, unless indicated otherwise in a credit line to the material. If material is not included in the article's Creative Commons licence and your intended use is not permitted by statutory regulation or exceeds the permitted use, you will need to obtain permission directly from the copyright holder. To view a copy of this licence, visit http://creativecommons.org/licenses/by/4.0/. 


\section{References}

1. Rizzoli P, Iuliano S, Weizenbaum E, Laws E (2016) Headache in patients with pituitary lesions: a longitudinal cohort study. Neurosurgery 78(3):316-323

2. Scheithauer BW, Gaffey TA, Lloyd RV, Sebo TJ, Kovacs KT, Horvath E et al (2006) Pathobiology of pituitary adenomas and carcinomas. Neurosurgery 59(2):341-353 (discussion 53)

3. Penn DL, Burke WT, Laws ER (2018) Management of non-functioning pituitary adenomas: surgery. Pituitary 21(2):145-153

4. Naganuma H, Satoh E, Nukui H (2002) Technical considerations of transsphenoidal removal of fibrous pituitary adenomas and evaluation of collagen content and subtype in the adenomas. Neurol Med Chir (Tokyo) 42(5):202-212 (discussion 13)

5. Snow RB, Lavyne MH, Lee BCP, Morgello S, Patterson RH (1986) Craniotomy versus trans-sphenoidal excision of large pituitary-tumors - the usefulness of magnetic-resonance-imaging in guiding the operative approach. Neurosurgery 19(1):59-64

6. Zada G, Du R, Laws ER Jr (2011) Defining the "edge of the envelope": patient selection in treating complex sellar-based neoplasms via transsphenoidal versus open craniotomy. J Neurosurg 114(2):286-300

7. Mahmoud OM, Tominaga A, Amatya VJ, Ohtaki M, Sugiyama K, Sakoguchi T et al (2011) Role of PROPELLER diffusionweighted imaging and apparent diffusion coefficient in the evaluation of pituitary adenomas. Eur J Radiol 80(2):412-417

8. Pierallini A, Caramia F, Falcone C, Tinelli E, Paonessa A, Ciddio $\mathrm{AB}$ et al (2006) Pituitary macroadenomas: preoperative evaluation of consistency with diffusion-weighted MR imaging-initial experience. Radiology 239(1):223-231

9. Snow RB, Johnson CE, Morgello S, Lavyne MH, Patterson RH $\mathrm{Jr}$ (1990) Is magnetic resonance imaging useful in guiding the operative approach to large pituitary tumors? Neurosurgery 26(5):801-803

10. Yamamoto J, Kakeda S, Shimajiri S, Takahashi M, Watanabe K, Kai Y et al (2014) Tumor consistency of pituitary macroadenomas: predictive analysis on the basis of imaging features with contrast-enhanced 3D FIESTA at 3T. AJNR Am J Neuroradiol 35(2):297-303
11. Sack I, Beierbach B, Hamhaber U, Klatt D, Braun J (2008) Noninvasive measurement of brain viscoelasticity using magnetic resonance elastography. NMR Biomed 21(3):265-271

12. Kruse SA, Rose GH, Glaser KJ, Manduca A, Felmlee JP, Jack CR Jr et al (2008) Magnetic resonance elastography of the brain. Neuroimage 39(1):231-237

13. Green MA, Bilston LE, Sinkus R (2008) In vivo brain viscoelastic properties measured by magnetic resonance elastography. NMR Biomed 21(7):755-764

14. Hughes JD, Fattahi N, Van Gompel J, Arani A, Ehman R, Huston J 3rd (2016) Magnetic resonance elastography detects tumoral consistency in pituitary macroadenomas. Pituitary 19(3):286-292

15. Jakobsson S, Olsson DS, Andersson E, Hallen T, Krabbe D, Olofsson AC et al (2020) Extended support within a person-centered practice after surgery for patients with pituitary tumors: protocol for a quasiexperimental study. JMIR Res Protoc 9(7):e17697

16. Le Bihan D, Ichikawa S, Motosugi U (2017) Diffusion and intravoxel incoherent motion mr imaging-based virtual elastography: a hypothesis-generating study in the liver. Radiology 285(2):609-619

17. Bahuleyan B, Raghuram L, Rajshekhar V, Chacko AG (2006) To assess the ability of MRI to predict consistency of pituitary macroadenomas. Br J Neurosurg 20(5):324-326

18. Thomas T, Gopalakrishna C, Thomas B, Nair S (2014) Evaluation of consistency of pituitary macroadenoma using diffusionweighted imaging in correlation with surgical findings. Neurosurg Quat 24(2):131-135

19. Alimohamadi M, Sanjari R, Mortazavi A, Shirani M, Moradi Tabriz H, Hadizadeh Kharazi H et al (2014) Predictive value of diffusion-weighted MRI for tumor consistency and resection rate of nonfunctional pituitary macroadenomas. Acta Neurochir (Wien) 156(12):2245-2252 (discussion 52)

Publisher's Note Springer Nature remains neutral with regard to jurisdictional claims in published maps and institutional affiliations. 